\title{
Prospects for Anesthesia in Day Surgery in Children
}

\author{
Nasibova Esmira* \\ Department of Pediatric Surgery, Azerbaijan Medical University, Azerbaijan
}

Submission: October 28, 2017; Published: November 27, 2017

*Corresponding author: Nasibova Esmira, Department of Pediatric Surgery of the Azerbaijan Medical University, Azerbaijan, Email: nasibova_esmira@mail.ru

\section{Introduction}

One of the urgent problems of modern pediatric surgery is the organization of high quality care for children in the hospital one day, as most of the planned operations with proper patient selection and application of minimally invasive procedures can be performed in a day surgery.

The main advantages of day surgery in children are:

A. Reduction of the children's stay in hospital;

B. Reduction of the psychological trauma in children because of the separation from their parents;

C. Early activation and checkout of patients;

D. Reduction of nosocomial infections.

The term one-day surgery involves hospitalization, surgery, awakening the immediate postoperative period and the patient in a statement the same day. The experience of creating a surgical hospital one day associated with the name James Nisaki. In 1909 he published an article in the British Medical Journal, which described the 8988 result of surgical procedures in children performed for 10 years in a children's hospital in Glasgow (UK). He showed that the comparable results of the cost of medical treatment in the hospital one day are 10 times less than in hospitals. And the first reaction of leading European magazines was extremely negative. But in the sixties of the XX century in the journal "Lancet" was first used the term "one day surgery", which became later the main, in the description of the surgery on an outpatient basis later there were other terms, in which the authors do not always put the same meaning.

An example of this is the term "ambulatory surgery". The terms "ambulatory surgery" and "day surgery" are very similar, but not identical. By first understanding the patient's stay in the hospital for only a few hours after surgery, followed by patronage supervision at home. When performing surgical interventions was widespread system of hospital one day. This system makes it possible to observe the patient until the next day, make the dressing, and write them under the supervision of the surgeon in the community. "One day surgery" reduces the time the child is located in the clinic, thereby reducing the risk of nosocomial infections, and iatrogenic complications, allows parents to actively participate in the postoperative period. Since the stress experienced by the child during his hospitalization is mainly due to, the fear of separation from parents, the one day surgery can reduce the psychological discomfort associated with it. Despite the fact that one day surgery performed for a long time, has not yet developed its clear standards. It is important to note the need for joint participation of the anesthesiologist and surgeon in the selection of children for surgical intervention in a one day surgery. Prior to the decision of surgical interventions in the hospital one day a doctor should make sure that there is no threat of deterioration of the child after the operation.

In the context of the one day surgery can be performed following surgical interventions and diagnostic procedures:

a) Hernia repair inguinal, femoral and umbilical hernias and hernia the white line of the abdomen;

b) Surgery for hydrocephalus shell eggs;

c) Circumcision;

d) Surgery for varicocele, spermatic cord cysts and epididymis, short frenulum foreskin, undescended testis;

e) Surgery for benign surface formations;

f) Excision of epithelial coccygeal;

g) Removal of polyps single anal canal, etc,

h) Gastroscopy, colonoscopy, bronchoscopy, MRI and CI.

Criteria for selection of patients for day surgery have recently become more liberal. Whereas before these operations were considered valid only in patients with class I and II ASA, how operate in many of the centers are in satisfactory condition at the class III patients on ASA. In severe co morbid conditions (morbid obesity, type, diabetes, asthma, myasthenia gravis) the decision should be individualized, taking into account the severity of the 
disease, the patient's condition and the nature of the operation. Our experience shows that in the preoperative examination of children in day surgery is not necessary, the appointment of excessive amounts of various routine laboratory examinations. Also, numerous studies have shown that with proper history and physical examination to identify the disease is easier than using a standard set of extensive tests.

Criteria for selection of patients for day surgery were divided us into three groups: non-medical, surgical and anesthetic.

\section{Non-Medical Criteria are}

Patients must mentally healthy;

I. Within 24 hours after surgery, the patient must be an adult (best mother), which can provide adequate surveillance of them;

II. Living conditions in which the patient will remain after the operation should be good enough, which will be followed hygiene rules;

III. The availability of operational telephone communication with the clinic, as well as the presence of the patient's cell or home phone;

IV. Transporting the patient from the hospital should be made private, not public transport, and this should be a short trip within one city and no more than one hour on the road;

V. The ability of parents to provide adequate care for a sick child.

\section{Surgical selection criteria}

a) Low volume of surgical intervention;

b) Duration of operation is not more than 2 hours;

c) The operation is not accompanied with greater blood loss;

d) Operation does not represent a potential risk to the patient's life;

e) No need for a specific post-operative care.

\section{Anaesthetic selection criteria}

We have identified absolute and relative criteria for operations under day surgery. Absolute contraindications include:

a) Premature, whose estimated age from conception less than 50 weeks (in some centers, 60 weeks);

b) Symptoms of apnea prematurity and bronchopulmonary dysplasia in the last 6 months;

c) Young children, whose brothers and sisters died from sudden infant death syndrome;

d) Children at high risk for ASA (class III-IV); e) Epilepsy, myasthenia gravis, malignant hyperthermia in history;

f) Polyvalent allergy to drugs.

In the above groups of patients at increased risk of postoperative apnea risk and they should be monitored for at least 24 hours after surgery. Relative contraindications include:
A. Uncomplicated congenital heart disease;
B. Epilepsy and asthma in remission;
C. Children from previous infections and rocket upper respiratory tract.

To summarize the criteria for selection of patients it must be concluded that the applied solution is always deeply personal, so the final verdict should be imposed the most experienced and qualified anesthesiologist.

One of the important moments in the day surgery in children is a inoperative preparation. Before anesthesia, a complete clinical and laboratory examination, as well as discussions with the parents. An indispensable condition should be voluntary consent of the parents to carry their child surgery in hospital one day. It should be legally formalized written consent for surgery. After that, the anesthesiologist should tell parents about the forum of pain relief. Explanations given during the pre-talk should be detailed and truthful. Extremely simple, but great detail is necessary to explain to parents the importance of the following points:

A. The need to exclude food or liquid before the operation (specify the terms of abstinence).

B. Consequences of failure of this action. If, even after the most thorough explanation to parents about the need to avoid eating before coming to the hospital, the doctor suspected the presence of a child's full stomach, the surgery should be postponed.

During preoperative discussions, in addition to history and examination of the patient, the anesthesiologist must make contact with the child and his parents. Often the fear of surgery is so great that persists even after it is finished. Therefore, the correct conduct interviews before surgery will prevent many unwanted physiological, social and emotional consequences. Terms of refraining from eating before surgery the following:

a) Solid food-up to 6 hours before surgery;

b) Breastfeeding-up to 4 hours before surgery;

c) Clear liquid-up to 2 hours before surgery.

The choice of anesthesia in day surgery in children is still an open and relevant. Currently, short term and little traumatic operation is increasingly performed in a hospital one day, which is reflected on the role of the anesthesiologist in the development of 
safe anesthesia techniques using new drugs. The main advantage of operations under day surgery is to reduce the risk of nosocomial infection, and from the standpoint of the patient and the parent operation in less impact on their personal lives. Quality, safety and efficiency-these are the main characteristics influencing the choice of anesthesia in a day surgery should provide rapid and smooth induction, good intraoperative analgesia and amnesia, muscle relaxation and enough short period of awakening without side effects.

In the context of the one day surgery it is very important to have safe and effective analgesics that have minimal side effects, however, the ideal anesthetic agent in pediatric anesthesia does not yet exist. In pediatric practice remains prevalent anesthesia with the use of inhaled anesthetics. But in recent years there has been a clear trend towards the implementation in practice of intravenous anesthesia kinds of anesthesia. Rapid induction and relatively short-term action, the ability to enhance the analgesic and hypnotic effects are undoubted advantages of the method of intravenous anesthesia. It should be mentioned that in pediatric patients it is not a full transition to the no inhalation anesthesia, and an increase in the proportion of different medications (analgesics, anesthetics, etc.) introduced by no inhalation, when combined anesthesia - inhalation and no inhalation.

No inhalation component of general anesthesia in children provides better analgesia and autonomic blockade. To this and against the background of low concentrations of inhaled or its absence various methods of anesthesia and regional anesthesia. Use as, a hypnotic anesthetic propofol based on the fact that it induces sleep quickly, supports off consciousness narcotic analgesics has fewer compared to other intravenous anesthetics, side effects. A very valuable quality of propofol is a good ability to cause muscle relaxation and suppress the laryngopharyngeal reflexes that can be used as a means to maintain the airway laryngeal mask. A combination of general and regional anesthesia on the basis of the concept of a "balanced" regional anesthesia is possible to achieve effective nociceptive unit is turned off while the child's consciousness. Complementing general anesthesia regional block is provided by the awakening of a painless and effective analgesia after operating and maintaining normal levels of ventilation.

The modern concept of central regional anesthesia is considering blockade day surgery as one of the most effective ways of interruption or inhibition of nociceptive information to the spinal level. Improving the efficiency of anesthesia at the present stage is largely due to the application of the concepts of "warning" and "multimodal" analgesia ("balanced" analgesia). The current, concept of "balanced regional anesthesia" is considering a regional blockade is not an alternative to general anesthesia, and as a complement to the latter, allowing to significantly reduce the concentration of both narcotic analgesics, inhalation and no inhalation anesthetics, and muscle relaxants.
The advantage of regional block is the fact that it provides adequate performance postoperative analgesia with preservation of consciousness, normal control ventilation and minimal risk of respiratory depression. Our numerous clinical studies (more than 1578 children) indicates that the central regional blockade in children are simple in execution, reliability and safety.

During regional blockade is necessary to take into account anatomical and physiological features of the child's body, which are the following:

i. In infant spinal cord (conus medullaris) ends at a lower level (L3) and reaches adult levels (L1) about the end of the first year of life. Therefore, spinal puncture for subarachnoid block in neonates and infants should only be performed at the level of L4-L5 or L5-S1 intervertebral spaces.

ii. Form of the aitch bone in infants more broad and flat than in adults. Access to the subarachnoid space of the sacral canal in newborns is more direct than in older children. The lower edge of the dural sac at the birth of projects on the level of S3-S4 and the second year of life gradually rises to the level of S2 (like an adult). The low dural sac creates a higher risk of subarachnoid puncture when performing caudal block in the newborn and infant.

iii. The epidural space if filled with infant's loose fatty tissue with a low content of fibrous fibers. In children older than 6-8 years, epidural fat is more densely packed significantly affect the distribution of local anesthetic solution.

iv. Vienna epidural space have no valve, therefore, accidental introduction of the anesthetic, the air in the epidural veins can cause almost instantaneous development of systemic toxik reactions.

v. In children weighing less than $15 \mathrm{~kg}$, the volume of cerebrospinal fluid per $\mathrm{kg}$ of body weight about twice $(4 \mathrm{ml} /$ $\mathrm{kg}$ ) tan in adults $(2 \mathrm{ml} / \mathrm{kg})$. This may partly explain the need for relatively high doses of local anesthetics in young children.

Hypotension (the result of sympathetic blockade) after technically correct, the central block is a very rare complication in children under 8 years (caudal, spinal and epidural block does not require a prior increase preload by volemic backups). Our experience shows that even in children older than 10 years, fluctuations in blood pressure do not exceed $10-20 \%$ of the initial level. Amond the factors explaining the high hemodynamic stability especially in infants and young children, include the immaturity of the sympathetic autonomous system, lower peripheral vascular resistance and a smaller fraction of the total volume of blood is sequestered in the lower extremities.

In our practice in the hospital one day, we often use the caudal anesthesia combined with general anesthesia. Caudal anesthesia is a method of a single bolus injection of a local anesthetic into a sacred space. This method is especially recommended for most 
traumatic operations in the lower third of the abdomen, perineum and the lower extremities (e.g. congenital malformations of the urethra, inguinal and umbilical hernia, cryptorchidism, varicocele, retroperitoneal tumor, anorectal surgery, trauma and orthopedic surgery of the lower libs). It is known that the dura mater ends at S3-S4 am birth and level S2 for only two years, remaining at this level in adults. This low position of the dural sac increases the risk spinal puncture when performing caudal block in infants. Therefore, when performing caudal anesthesia in infants technical details of the implementation unit should pay particular attention.

Caudal space is the lower portion of the epidural. The sacrum is a triangle, which consists of five fused sacral vertebrae (S1-S5). As you know, the sacrum is the cartilage structure in infants and young children. Finally, the process of ossification is completed closer to the 25 years of life. Sometimes it may be cleft vertebral arches at the sacrum, which increases the risk of puncture of the dura mater when the caudal anesthesia. In other cases, in contrast, found their complete fusion, and then in the absence of sacral holes, caudal blockade becomes impossible. Sacral hole is a defect bottom of the rear wall of the sacrum plate formed S5 and S4. There are significant variations in the structure of the sacral canal. The sacral canal is a continuation of the lumbar epidural space, and ends with the sacred hole. Volume sacral canal in adults varies greatly and includes:

Terminal of the dura mater at the level of S1-S3;

i. The five sacral nerves and coccygeal nerves that farm the cauda equine. Sacral epidural veins usually disappear at S4, but can be spread across all channels, so there is a risk of perforation of the needle or cathter;

ii. Fulum terminale-terminal portion of the spinal cord, does not contain nerves that go through the sacral canal and attached to the rear of the coccyx;

iii. Epidural fat, soft consistent varies from children to adults fibrous. This difference causes predictable spread of the anesthesia in children and adults at the unpredictable. The peculiarity of sacred space is that it is free communicates with perineural space of the spinal nerves beginning of the lumbosacral plexus. Because of this with the help of the caudal block is possible to achieve adequate analgesia, organs below the navel.

\section{Technique Caudal Block in Children}

Caudal block in children is carried out in the supine position on the left side a little given to his chest hips and knees. It should be separately emphasized that in pediatric anesthesiology all kinds of regional block, including caudal epidural anesthesia is performed in patients already in a state of anesthesia. At the caudal anesthesia particular importance is the correct location of the needle in the caudal space, as there is a risk of mechanical damage to the nerves of the sacral canal with the development of neurological complications. Furthermore, in case of improper needle placement is a risk of contact of the anesthetic into the subdural space development total spinal unit (deep hypotension, loss of consciousness, protective reflexes, cardiac and respiration), or into the lumen of the vessel with the advent of toxic complications, seizures, arrhythmias, up to ventricular fibrillation. Because there are significant anatomical variations, the definition of bony landmarks is optional and is the key to success.

It is important to establish the midline of the sacrum as the variability of expression of horns sacrum can cause problems puncture. In practice, we define the following guidelines, which are palpated at the level below the last sacral vertebra: is the sacrococcygeal membrane covers the triangular space which is bordered on either side with a sacred horns, sacrum and coccyx. Palpation of the membrane voltage provides a tactile sensation similar to that of palpation of large fontanels in infants. Puncture point generally located in the center of this triangle. Puncture technique is quite simple. The skin in the projection of the sacrum and the puncture site is treated with antiseptic, sterile sheets and hiding the use of sterile gloves is strictly necessary. First, the index finger of his left hand palpates hiatus sacralis, with the other hand takes the needle (as a pen), slice it is directed to the side, and then the needle is inserted perpendicular to the midline sacococcygeal membrane. After puncture of the skin and subcutaneous tissue is necessary to set the direction of the needle $30-40^{\circ}$ with respect to the skin and continue to slowly move in the cranial direction. When passing through the sacrococcygeal membrane there is a feeling of failure, similar to those with spinal, epidural puncture during the yellow ligament. Then, after such a tactile identification of the caudal epidural space, the needle moves another 2-3 $\mathrm{mm}$ and this is done to achieve the full confidence of finding just cut the tip of the needle in the sacral canal. It should be noted that the depth location caudal space depends, on the thickness of the tissues that cover the sacrococcygeal membrane and varies depending on the age and weight of the patient.

This is the classic technique of caudal block in our practice we adhere to other vehicles of the caudal block, so-called "technology without turning the needle" ("the no turn technique"), which is to puncture sacrococcygeal ligament at an angle of 60 degrees with no advancement of the needle in the sacral canal. Proposed and studied by us, this technique allows a caudal block $100 \%$ of its applications, in contrast to the classical, where the success rate was $85 \%$ of punctures. To puncture the caudal space we used conventional or intramuscular needle or needle size 19-21G "Epican". Adequate internal diameter of the needle allows you to quickly see the reflux of blood or cerebrospinal fluid to the pavilion of the needle when it enters the lumen of the vessel or accidental puncture of the dural sac. On the other hand, a sufficient outer diameter makes the rigid needle and allows passage clearly feels the needle through the membrane. The length of the needle cannot exceed 3-4 centimeters, as the distance from the skin to the caudal epidural space does not exceed 20 millimeters. Because of the 
dura mater is too dangerous to use a long needle. After entering the sacred space is needed for 10-15 seconds, carefully inspect the pavilion of the needle and make sure there is no free flow of blood or cerebrospinal fluid (puncture of the vessel or the dural sac).

When the blood in the pavilion of the needle, the latter is extracted and executed retried new needle. Then the needle hook the fingers of the left hand and the base of the palm resting on the surface of the sacrum carefully fix its position throughout the time of administration of the local anesthetic. The other hand is connected with the preparation syringe, perform the first aspiration test and begin the introduction of the solution. Moreover, aspirate carried out every $2 \mathrm{ml}$ injection. If the suction is not received blood or cerebrospinal fluid, the test was carried out administering the close of local anesthetic, then carefully for 30-40 seconds evaluated hemodynamics and electrocardiogram. In the absence of hemodynamic and ECG changes were introduced the remaining volume of local anesthetic for 60-90 seconds, as too rapid injection resulted in increased intracranial pressure, slow introduction-lateralization unit.

The rate of introduction is also an important factor. Fully local anesthetic must be administered for 60-90 seconds, which corresponds to the rate of introduction of 0,023-0,033 ml/sec. Faster injection speed create the danger of excessive spreading of the solution in the cranial solution, which can lead to higher level of blockade. Moreover, rapid introduction of a solution leads to a change in pressure in the epidural space, respectively, and intracranial, which is accompanied by a transient hypotension. On the other hand too slow injection can create the conditions for the lateralization of the block. Furthermore, when the drug is too slow introduction needle remains in the sacral canal, which increases the risk of displacement. After completion of the administration of the anesthetic needle is pulled out of the sacral canal and the child fit into the desired position for surgery. From time to time the injection into the caudal space and before step (latent period) independent of the local anesthetic used and ranged from 10 to 20 minutes. It has been observed that children up to 5-6 years, the latency of local anesthetics is somewhat less than in older patients.

The inability to puncture the caudal space due to anatomical features was the basis for the refusal of the caudal blockade and the transition to alternative methods of anesthesia. Palpable in these patients there were no standard guidelines in the form of cornu sacralis and base of the coccyx, this could be due to the complete or partial closure of the hiatus sacralis.

\section{Physiology Caudal Anesthesia}

The physiological effect of caudal blockade is almost identical to the action on the lumbar epidural level. Intensity of the blockade depends on the achieved level of anesthesia, which in turn is determined by the amount of anesthetic. Theoretically, entering a very large amount of anesthetic in the caudal access you can achieve the blockade average breast and even the upper thoracic segments. In such a case, a physiological effect indistinguishable from epidural anesthesia at the lumbar level. The extreme variability of the anatomy of the sacrum - especially the configuration of the sacral canal - virtually eliminates the possibility of high epidural sacral access.

\section{The Technique of Anesthesia in Patients Operated Under Day Surgery}

Caudal blockade was carried out only in a superficial general anesthesia, after intubation and before the operation. The technique of general anesthesia in all cases remained standard. In children older than one year the introduction of propofol sedation was carried out at the rate of $2 \mathrm{mg} / \mathrm{kg}$ in the preoperative set of tableware in the presence of parents. After premedication sleepy children are separated from parents and enters the operating room. Further, the total induction in children older than 12 months was conducted propofol $2 \mathrm{mg} / \mathrm{kg}$. Tracheal intubation was performed after intravenous administration of no depolarizing muscle relaxant action esmeron rate of $0,6 \mathrm{mg} / \mathrm{kg}$. In children younger than one year of induction of general anesthesia was performed by inhalation sevorane about 6 vol \%.

After a full sleep and tracheal intubation was performed after intravenous administration esmeron rate of $0,6 \mathrm{mg} / \mathrm{kg}$. Maintenance of anesthesia in all groups of children performed using isoflurane about 0,6 vol.\%-0,8 vol.\% or sevorane 2 vol \%. After intubation the patients underwent caudal block at the position on the left side. With effective caudal block any one patient didn't require the introduction of a narcotic analgesic and a muscle relaxant. All patients carried on volume controlled ventilation mode IPPV with the ventilation parameters, ensuring the maintenance of ETCO2 within 35-40 mm Hg. Mandatory monitoring included: indicators of non-invasive blood pressure, ECG, pulse oximetry and capnometer. Performance recorded using the monitor from the start of induction of anesthesia before the full awakening of the patient after surgery. In all patients, infusion therapy was performed for all operations in the amounts corresponding to the weight of the child and the nature of the surgery.

\section{Complications observed during caudal block}

Cuadal blockade is one of the safest methods of regional anesthesia with constant respect for the rules of her safe conduct. However, complications in its performance as possible and generally in accordance with epidural anesthesia complications. Caudal anesthesia complications include:

a) Intravascular drug leads to development of systemic toxic reaction, especially when using bupivacaine. Help includes treatment of systemic toxic reacton on the general principles (fighting crams, ventilation and hemodynamic support).

b) Intrathecal administration of the anesthetic due to dural puncture leads to immediate and inevitable development 
of total spinal block. Treatment includes respiratory and hemodynamic support to all functions.

c) Rectal perforation occurs when uncontrolled introduction of a needle. Dangerous in itself, but even more dangerously getting contaminated needle into the epidural space when it is withdrawn.

d) Damage periosteum occurs when applying it scratches sharp tip of the needle, usually accompanied by very perceptible pain which may persist for several weeks. In the latter case, a purpose of nonsteroidal anti-inflammatory drugs and physiotherapy.

e) Retention of urine is relatively frequent and requires catheterization.

f) Epidural hematoma is rare, usually during caudal blockade in patients receiving anticoagulants. Treatment with surgical.

g) The intraosseous injection is also rare, but can lead to the development of systemic toxic reactions to local anesthetics. The hallmark of development of the reaction is not instantaneous as when administered intravenously, and after a few minutes. h) Hypertension occurs when the forced administration of anesthetic solution and is associated with a response to compression of the nerve, located in the sacral canal. Usually this complication resolve spontaneously and prevents a slow injection.

\section{The Choice of Drug for Caudal Anesthesia}

It is advisable to choose a drug with a long duration of action and minimal side effects. Formulations for normal introduction of the vials should be for a single use without the addition of preservatives. Proposed set of recommendations for the calculation of doses of local anesthetics, which very quite widely:

I. Armitage recommends $0.25 \%$ bupivacaine administered in a dose of $0.5 \mathrm{ml} / \mathrm{kg}$ for lyumbosacral block, $1 \mathrm{ml} / \mathrm{kg}$ - for the lyumbar-thoracic unit and $1.25 \mathrm{ml} / \mathrm{kg}$ for the block at the middle level. The maximum dose of bupivacaine should not exceed $20 \mathrm{ml}$. For large volumes, he recommends adding one part of $0.9 \% \mathrm{NaCl}$ to the three parts of the local anesthetic for $0.19 \%$ solution.

II. Scott calculates the dose for age and weight of the child. If the child is of average weight for their age, the two numbers are same. And if the child more weight, you should use the figure, based on the age, in order to avoid overdose (Table 1).

Table 1: Calculations of the dose for age and weight of the child by Scott.

\begin{tabular}{|c|c|c|c|}
\hline Weight (kg) & Age (years) & $\begin{array}{c}\text { A dose of } \mathbf{0 . 2 5} \text { \% bupivacaine } \\
\text { (ml) for the block to Th12 }\end{array}$ & $\begin{array}{c}\text { A dose of 0.25 \% bupivacaine } \\
\text { (ml) for the block to Th7 }\end{array}$ \\
\hline 12.5 & 2 & 4 & 6 \\
\hline 15 & 3 & 5 & 7.5 \\
\hline 16 & 4 & 5.5 & 8 \\
\hline 17.5 & 5 & 6 & 9 \\
\hline 20 & 6 & 7 & 10.5 \\
\hline 22.5 & 7 & 8 & 12 \\
\hline 25 & 8 & 9 & 13.5 \\
\hline 30 & 9 & 10 & 15 \\
\hline
\end{tabular}

Lower doses recommended Scott, designed to effect analgesia, whereas as relatively high doses proposed Armitage, provide anesthesia in adults, the use of $20-30 \mathrm{ml}$ for anesthesia at the lower half of the abdomen and $15-20 \mathrm{ml}$ - to the blockade at the level of the perineum and lower extremities. As a result of many years of experience and numerous application caudal anesthesia titration method we determined the dose of drugs, which are displayed in the Table 2 .

Table 2: Caudal anesthesia titration method for determination of the dose of drugs.

\begin{tabular}{|c|c|c|c|c|}
\hline Preparation & \% & Dose (mg/kg) & Time (min) & Anesthesia(min) \\
\hline Prilocain & 1 & 7 & 15 & 200 \\
\hline Bupivacain & 0.25 & 2 & 12 & 550 \\
\hline Naropin & 0.2 & 2 & 10 & 520 \\
\hline Levobupivacain & 0.25 & 1 & 8.5 & 620 \\
\hline
\end{tabular}


Our experience of caudal anesthesia in children in day surgery led to the following conclusions:

i. Effective caudal block combined with general anesthesia for surgical interventions in children contributed to the complete abandonment of the use of narcotic analgesics. ii. Caudal anesthesia provides effective protection against nociceptive surgical interventions in children below the navel on the background surface of general anesthesia.

iii. As a result of the study and application technology caudal block anesthesia success rate was rather high (>95 \%).

\section{Your next submission with Juniper Publishers} will reach you the below assets

- Quality Editorial service

- Swift Peer Review

- Reprints availability

- E-prints Service

- Manuscript Podcast for convenient understanding

- Global attainment for your research

- Manuscript accessibility in different formats ( Pdf, E-pub, Full Text, Audio)

- Unceasing customer service

Track the below URL for one-step submission https://juniperpublishers.com/online-submission.php 